\title{
Abundancia relativa, diversidad y patrones de actividad de mamíferos terrestres medianos y grandes, sendero Pájaro Sombrilla, Reserva Biológica Alberto Manuel Brenes, Alajuela, Costa Rica
}

\author{
Relative abundance, diversity and activity patterns in the medium and large land mammals in Pájaro \\ Sombrilla trail, Alberto Manuel Brenes Biological Reserve, Alajuela, Costa Rica
}

\author{
Ronald Sánchez Porras ${ }^{1}$ \\ Liz Brenes Cambronero² \\ Kevin Chavarría Esquivel ${ }^{3}$ \\ Yeison Mejías Vásquez ${ }^{4}$
}

Fecha de recepción: 24-3-20

Fecha de aceptación: 16-3-21

\begin{abstract}
Resumen
Con el uso de cámaras trampa se registraron y analizaron las poblaciones de mamíferos terrestres medianos y grandes presentes en el sendero Pájaro Sombrilla, dentro de la Reserva Biológica Alberto Manuel Brenes, Alajuela, Costa Rica. La investigación se realizó desde agosto de 2017 a septiembre de 2018, y abarcó las temporadas seca y lluviosa. Con un esfuerzo de muestreo de 1584 días-trampa, se captaron 21 especies de mamíferos pertenecientes a 7 órdenes y 15 familias. Se registraron 10 especies de carnívoros, este fue el orden más representado. El estudio mostró una riqueza específica de $\mathrm{S}=21$. El índice de Margalef obtuvo una diversidad alta (seca Dmg=2.47, lluviosa Dmg= 3.09), además el índice de Pielou indicó que en ambas temporadas se mantuvo abundancia de media a alta (seca e $=0.69$, lluviosa e $=0.69$ ). El índice de Shannon-Wiener mostró una diversidad moderada $\left(H^{\prime}=2.04\right.$; seca $H^{\prime}=1.86$, lluviosa $H^{\prime}=2.03$ ). La prueba de Hutchenson reflejó que el índice de Shannon-Wiener es significativo, así la época lluviosa se diferencia de la época seca ( $\mathrm{t=1.69;} \mathrm{p}=0.05$; $\mathrm{gl}=619)$. Se capturó un 95.45\% de las especies terrestres de mamíferos medianos y grandes existentes en el sendero Pájaro Sombrilla, según los resultados de la curva de acumulación de especies. En relación con el índice de abundancia relativa calculado, las especies más abundantes fueron Pecari tajacu (IAR=18.50), Leopardus pardalis (IAR=2.21), Puma concolor ( $I A R=2.15$ ) y la familia Didelphidae (IAR=2.78). En cuanto a los patrones de actividad, P. tajacu presentó actividad diurna (6:00-20:00). L. pardalis tuvo actividad catameral con dos picos máximos (6:00-8:00 y el otro de 22:00 - 24:00), P. concolor presentó actividad catameral con máximos de actividad a las (14:00-16:00 y 22:00-24:00). Los datos que se presentan en esta investigación pueden ser útiles para la creación de programas de manejo y conservación de la Reserva, sus especies y las interacciones entre estas.
\end{abstract}

Palabras clave: biodiversidad, cámaras-trampa, mamíferos, patrones de actividad, Reserva Biológica Alberto Manuel Brenes.

\footnotetext{
1 Magíster en Manejo de Fauna Silvestre. Docente investigador de la Sección de Biología, Programa de Investigaciones en Manejo Sostenible de los Recursos Naturales, Universidad de Costa Rica, Sede de Occidente, San Ramón, Costa Rica. Correo electrónico: ronald.rsr@gmail.com

2 Magíster en Biología. Docente investigadora de la Sección de Biología, Programa de Investigaciones en Manejo Sostenible de los Recursos Naturales, Universidad de Costa Rica, Sede de Occidente, San Ramón, Costa Rica. Correo electrónico: 1lmbrenes@hotmail.com

3 Estudiante Maestría en Gestión del Recurso Hídrico para Latinoamérica y el Caribe, Gestor de los Recursos Naturales, Universidad de Costa Rica, Sede de Rodrigo Facio, Costa Rica. Correo electrónico: kevinchaes@hotmail.com

4 Estudiante de la Carrera de Gestión de los Recursos Naturales, Universidad de Costa Rica, Sede de Occidente, San Ramón, Costa Rica. Correo electrónico: yeisonyx15@hotmail.com
} 


\begin{abstract}
The use of trap cameras allow us to recorded and analyzed the populations of medium and large terrestrial mammals present in the Pajaro Sombrilla trail in Alberto Manuel Brenes Biological Reserve, Alajuela, Costa Rica. The research was from August 2017 to September 2018, and covered the dry and rainy seasons. With a sampling effort of 1584 trap days, 21 species of mammals belonging to 7 orders and 15 families were captured. Ten species of carnivores were registered, this was the most represented order. The study showed a specific richness of $S=21$. The Margalef index obtained a high diversity (dry Dmg $=2.47$, rainy Dmg = 3.09), in addition the Pielou index indicated that in both seasons abundance was maintained from medium to high (dry e $=$ o.69, rainy e = 0.69). The Shannon-Wiener index showed a moderate diversity $\left(\mathrm{H}^{\prime}=2.04\right.$; dry $\mathrm{H}^{\prime}=1.86$, rainy $\left.\mathrm{H}^{\prime}=2.03\right)$. The Hutchenson test showed that the Shannon-Wiener index is significant, so the rainy season differs from the dry season ( $\mathrm{t}=1.69$; $\mathrm{p}=0.05 ; \mathrm{gl}=619$ ). 95.45\% of the terrestrial species of medium and large mammals were captured on the Pajaro Sombrilla trail, according to the results of the species accumulation curve. In relation to the calculated relative abundance index, the most abundant species were Pecari tajacu (IAR = 18.50), Leopardus pardalis (IAR = 2.21), Puma concolor (IAR = 2.15) and the Didelphidae family (IAR = 2.78). Concerning to activity patterns, $P$. tajacu presented daytime activity (6: oo-20: oo). L. pardalis had catameral activity with two maximum peaks (6: oo-8: oo and the other from 22:00 - 24:00), P. concolor presented catameral activity with maximum activity at (14: 00-16: oo and 22: 00-24: oo). The data presented in this investigation can be useful for the creation of management and conservation programs for the Reserve, its species and the interactions between them.
\end{abstract}

Keywords: biodiversity, camera-traps , mammals, activity patterns, Reserva Biológica Alberto Manuel Brenes

\section{Introducción}

La toma de decisiones efectiva en el manejo de un área protegida, debe estar basada en la dinámica del bosque y en la biodiversidad contenida en el ecosistema (Cifuentes, Izurieta \& Faria, 2000). Los mamíferos grandes pueden ser indicadores de condiciones ecológicas y del estado de conservación del ambiente (Rumiz, 2010).

Las cadenas alimenticias en los ecosistemas deben ser conocidas, una manera es mediante la investigación de los mamíferos grandes y medianos, ya que estas, pueden arrojar pistas para conocer el estado de salud de un bosque. Una investigación realizada por Carrillo \& Sáenz (2013) menciona que los grandes felinos, como el jaguar y el puma (Puma concolor), y sus presas, entre ellas, el chancho de monte (Tayassu pecari), la danta (Tapirus bairdii) y el cabro de monte (Mazama americana) están muy relacionados con los bosques primarios. Además, Cortez-Marcial (2009) explica que algunas especies medianas como Leopardus pardalis y Pecari tajacu, son consideradas como indicadoras de ambientes conservados.
La Reserva Biológica Alberto Manuel Brenes cuenta con un bosque tropical húmedo premontano el cual presenta la particularidad de poseer mamíferos grandes como Tapirus bairdii, Panthera onca, Puma concolor, Leopardus pardalis, además de algunos mamíferos medianos como Dasypus novemcinctus, Pecari tajacu, Nasua narica, Cuniculus paca, los cuales permiten comprender las bases del comportamiento ecosistémico en estos ambientes particulares. Estas especies tienen funciones ecosistémicas importantes como la depredación, dispersión de semillas, competencia inter e intraespecífica y mutualismo, que son determinantes en la conservación de estos bosques (Rojas-Martínez \& Moreno, 2014).

Dentro de ese contexto ecosistémico, el conocimiento es vital para la comprensión de la dinámica del bosque, su mantenimiento y manejo. De ahí la importancia de estudios con cámaras trampas que han resultado ser la técnica más efectiva para realizar estudios de la abundancia relativa, diversidad y patrones de actividad de mamíferos por su capacidad no invasiva que provee datos confiables durante las 24 horas del día (Díaz-Pulido \& Payán, 2012; Artavia, 2015). 
Así, el objetivo del presente trabajo fue analizar la abundancia relativa, diversidad y patrones de actividad en mamíferos terrestres medianos y grandes en el sector del sendero Pájaro Sombrilla de la Reserva Biológica Alberto Manuel Brenes, Alajuela, Costa Rica.

\section{Materiales y métodos}

Área de estudio: La investigación se realizó en el Sendero Pájaro Sombrilla (SPS), dentro de la ReBAMB, entre las coordenadas $84^{\circ} 35^{\prime} 47.67^{\prime \prime} \mathrm{O} 10^{\circ} 13^{\prime} 8.62$ " N y $84^{\circ} 35^{\prime} 54.06$ ”O $10^{\circ} 13^{\prime 2} 1.36^{\prime \prime} \mathrm{N}$. SPS, mide 617 metros de longitud y está a una altitud máxima de $1035 \mathrm{msnm}$. Este sendero se localiza en la zona de vida bosque tropical húmedo de premontano (Fig. 1).

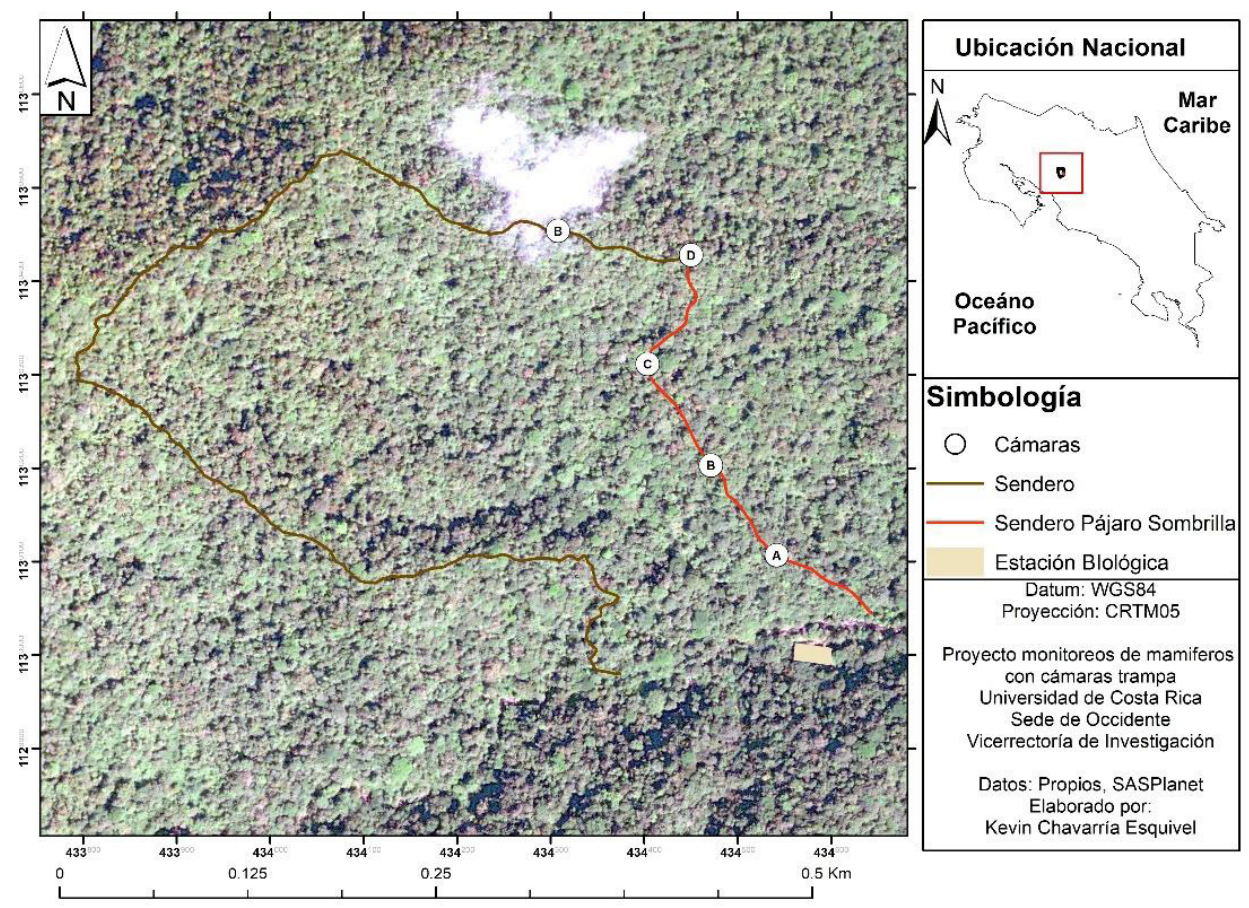

Fig. 1. Ubicación de las cámaras-trampa sobre el sendero Pájaro Sombrilla, ReBAMB (agosto 2017 a septiembre 2018), disposición espacial de A, B, C D. La cámara B se reubicó por daños en sendero por tormenta tropical Nate.

Diseño de muestreo: Este trabajo se llevó a cabo de agosto 2017 a septiembre 2018, por medio del uso de cámaras trampa y comprendió tanto la estación seca (enero-abril) como la lluviosa (diciembre-mayo), con base en los datos de la estación meteorológica CIGEFI (2018) (Sánchez et al., 2019). Se ubicaron cuatro estaciones de muestreo (A, B, C y D) con una cámara-trampa digital (Bushnell Natureview Cam HD 119739®) en cada una, a una altura de 40-6o cm del nivel del suelo y con una separación de 100 a 150 m, una de otra. Debido a la tormenta tropical Nate y la caída de árboles, fue necesario trasladar la cámara de la estación B, 100 metros después de la cámara D (Fig. 1). Se programaron las cámaras-trampa con el objeto de capturar videos con una duración de 30 segundos, cada 5 . El 28 de cada mes se recogieron los datos para un total de 14 tomas. Se tomaron en cuenta los parámetros establecidos por Arita \& Figueroa (1999); Ceballos \& Oliva (2005), para definir las tallas de mamíferos medianos y grandes. 
Abundancia relativa de especies: En cuanto a los datos de video-trampeo se delimitaron las siguientes pautas para definirlos como registros independientes: a) videos consecutivos de diferentes individuos, b) videos consecutivos de la misma especie, separadas por 24 horas (esto se empleó, si no se podía diferenciar al individuo capturado en el video), c) videos no consecutivos de la misma especie, (Medellín et al., 2006; Lira-Torres \& Briones-Salas, 2011; Monroy-Vilchis et al., 2011; LiraTorres \& Briones-Salas, 2012). Para esta investigación, se tomó en cuenta la clasificación taxonómica máxima identificable para cada registro (Sánchez, Brenes, Chavarría \& Mejías, 2019).

El índice de abundancia (IAR), se basa en la utilizada por Azuara (2005), Cartín \& Carrillo (2017), Jenks et al. (2011), Lira-Torres \& Briones-Salas (2012), Maffei, Cuellar \& Noss (2002) y Sanderson (2004), en el cual el IAR = $(\mathrm{C} / \mathrm{EM})^{\star} 100$, donde $\mathrm{C}$ es el número de capturas y EM, el esfuerzo de muestreo, calculado como el número de cámaras multiplicado por los días de muestreo hábiles de las cámaras, estacional o total (Sánchez et al., 2019).

Diversidad de especies: Para medir la diversidad de mamíferos del SPS, se emplearon:

a) El índice de Riqueza Específica, para cuantificar el número total de especies obtenidas por un censo de la comunidad determinada (Moreno, 2001).

b) El índice de Margalef, para calcular la diversidad numérica de individuos de diferentes especies, basados en el número de individuos existentes en una muestra analizada (Margalef, 1958; Moreno, 2001)

c) El índice de Equidad de Pielou, que establece una medición de la proporción de la biodiversidad observada, en relación con la máxima biodiversidad esperada (Moreno, 2001; Pielou, 1966).

d) El Índice de Shannon Wiener (Shannon \& Weaver, 1948; Moreno, 2001), fue sometido a dos muestras separadas, por estación lluviosa y seca, para conocer la biodiversidad en ambas.
Para saber si los cálculos del índice de Shannon Wiener son significativos, se usó la prueba 't' de Hutchenson con el objetivo de probar la igualdad de muestras desiguales (Hutchenson, 1970; Moreno, 2001).

El programa SurfStat (2018), en prueba de dos colas, empleó los grados de libertad y el valor de ' $t$ ' calculado para hallar el valor de 't' estadístico y probar la igualdad de las muestras.

Curva de Acumulación de Especies: el éxito de muestreo y el alcance del sitio sobre nuevas especies se determinó con el uso de la curva de acumulación de especies con los modelos de Clench (Soberón \& Llorente, 1993) y el modelo de acumulación de especies del paquete Vegan en lenguaje R, en el software RStudio (Shimwell, 1971; Oksanen et al., 2018).

Los datos fueron aleatorizados a 100 permutaciones en los programas RStudio (R Development Core Team, 2008) y EstimateS v.9.1.o (Colwell, 2006). Para las dos pruebas, los parámetros a y b de la curva se ajustaron con el programa Statistica 13.3 (Tibco, 2018), y se usó el algoritmo de Simplex \& Quasi-Newton, por su capacidad de minimizar la pérdida asociada a errores, mientras se estiman los parámetros (Jiménez-Valverde \& Hortal, 2003).

Además, se utilizaron fórmulas para determinar estadísticamente el esfuerzo de muestreo necesario para alcanzar una determinada proporción de la fauna, el porcentaje de especies registradas y la cantidad de especies que no se registraron (Soberón \& Llorente, 1993; Jiménez-Valverde \& Hortal, 2003).

Patrones de actividad: se determinaron los patrones de actividad al analizar la hora de las capturas totales de las especies independientes que obtuvieron más de 11 registros (totales, en época seca y época lluviosa) (Maffei, et al., 2002; Monroy et al., 2009; Lira-Torres \& BrionesSalas 2012), divididas según los datos de la estación meteorológica de la Universidad de Costa Rica (CIGEFI, 2018), donde los registros se ordenaron en intervalos de 2 horas y se clasificaron por horarios: diurnos (8:00 a 18:0o horas), nocturnos (20:00 a 6:0o horas), crepúsculo 
matutino (6:00 a 8:0o horas) y crepúsculo vespertino (18:00 a 20:00 horas)(Maffei et al., 2002; Monroy et al., 2011; Cortés \& Briones, 2014).

\section{Resultados}

Abundancia relativa: Las especies con mayor abundancia relativa fueron $P$. tajacu (IAR=18.50. $\mathrm{r}=293$ ), seguida de $N$. narica $(\mathrm{IAR}=4.04 . \mathrm{r}=64)$ y la familia Didelphidae $(\mathrm{IAR}=2.78 . \mathrm{r}=44)$. Las especies menos abundantes fueron
M. temama, Bassaricyon gabbi y Canis latrans con el mismo índice de abundancia relativa (IAR $=0.06, r=1$ ) (Cuadro 1).

Algunas especies como D. novemcinctus presentó una marcada diferencia en el IAR de la época lluviosa (IAR $=1.81$ ) versus la época seca, donde no hubo registros (Cuadro 1 y 2). Sin embargo, esta zona presenta solo 4 meses de época seca, de enero a abril.

\section{CUADRO 1}

Índice de Abundancia Relativa (IAR) de las especies registradas, sendero Pájaro Sombrilla, ReBAMB

\begin{tabular}{|c|c|c|c|c|c|}
\hline Orden & Familia & Especie & $\begin{array}{l}\text { IAR seco } \\
(480)^{*}\end{array}$ & $\begin{array}{l}\text { IAR } \\
\text { lluvioso } \\
(1104)^{*}\end{array}$ & $\begin{array}{l}\text { IAR total } \\
(1584)^{\star}\end{array}$ \\
\hline Carnivora & Procyonidae & Bassarycion gabbi & 0.21 & - & 0.06 \\
\hline Carnivora & Canidae & Canis latrans & - & 0.09 & 0.06 \\
\hline Carnivora & Mephidae & Conepatus semistriatus & 0.62 & 0.27 & 0.38 \\
\hline Rodentia & Cuniculidae & Cuniculus paca & $3 \cdot 54$ & 1.18 & 1.89 \\
\hline Rodentia & Dasyproctidae & Dasyprocta punctata & - & 0.18 & 0.13 \\
\hline Cingulata & Dasypodidae & Dasypus novemcinctus & - & 1.81 & 1.26 \\
\hline Didelphimorphia & Didelphidae & Didelphidae + & 5.21 & 1.72 & 2.78 \\
\hline Carnivora & Mustelidae & Eira barbara & 0.63 & 0.45 & 0.50 \\
\hline Carnivora & Felidae & Herpailurus yagouaroundi & 1.46 & 0.09 & 0.50 \\
\hline Carnivora & Felidae & Leopardus pardalis & 3.13 & 1.81 & 2.21 \\
\hline Carnivora & Felidae & Leopardus wiedii & 1.04 & 2.17 & 1.83 \\
\hline Artiodactyla & Cervidae & Mazama temama & 0.21 & - & 0.06 \\
\hline Rodentia & Sciuridae & Microsciurus alfari & - & 0.36 & 0.25 \\
\hline Carnivora & Mustelidae & Mustela frenata & 0.21 & 0.27 & 0.25 \\
\hline Carnivora & Procyonidae & Nassua narica & 5.63 & $3 \cdot 35$ & 4.04 \\
\hline Artiodactyla & Tajassuidae & Pecari tajacu & 28.96 & 13.95 & 18.50 \\
\hline Carnivora & Felidae & Puma concolor & $3 \cdot 54$ & 1.54 & 2.15 \\
\hline Rodentia & Sciuridae & Sciurus granatensis & - & 0.18 & 0.13 \\
\hline Rodentia & Sciuridae & Sciurus variegatoides & 2.05 & 1.36 & 1.70 \\
\hline Lagomorpha & Leporidae & Sylvilagus floridanus & - & 0.45 & 0.32 \\
\hline Pilosa & Myrmecophagidae & Tamandua mexicana & 0.83 & 0.09 & 0.32 \\
\hline
\end{tabular}

* Esfuerzo de muestreo.

+ Distinción taxonómica hasta familia (Didelphidae). 
Diversidad de especies: se realizó un muestreo de 1584 días-trampa, con cuatro cámaras trampa funcionales y se contabilizaron un total de 624 registros independientes. Además, se observó un total de 20 especies de mamíferos terrestres medianos y grandes identificables, que se dividen en 7 órdenes y 15 familias y un registro identificable hasta familia (Didelphidae). El orden dominante fue carnívora con 10 especies y el artiodáctila con mayor número de registros individuales (Cuadro 1).
Dentro de las especies registradas estaban $L$. wiedii, $L$. pardalis, $P$. concolor $y H$. yaguaroundi, que se encuentra dentro del apéndice 1 del CITES. Además L. pardalis que está en categoría casi amenazado y el $M$. temama que aparece con datos deficientes (IUCN, 2016) (Cuadro 2).

Por primera vez con registro fotográfico o prueba de video, se evidenció la presencia de S. floridanus, B. gabbi, C. latrans, S. granatensis y T. mexicana (Fig. 2).
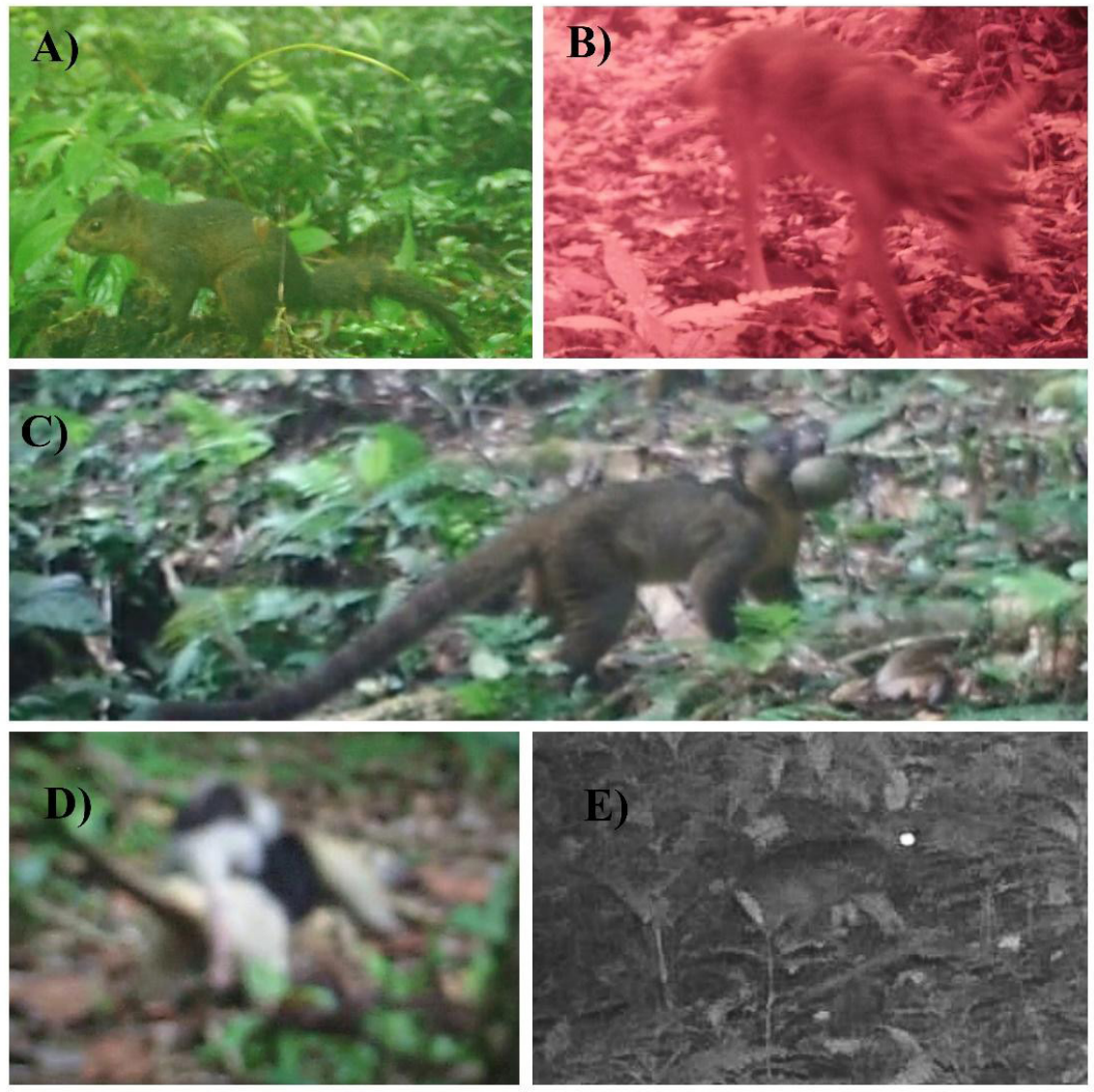

Fig. 2. Capturas fotográficas del S. granatensis (A), C. latrans (B), B. gabbi (C), T. mexicana (D) y S. floridanus (E), sendero Pájaro Sombrilla, ReBAMB.

Ocho especies no fueron registradas durante la estación seca: $C$. latrans, $D$. punctata, $D$. novemcinctus, M. temama, $M$. frenata y $M$. alfari, S. floridanus y S. granatensis. Mientras que, durante la estación lluviosa las cámaras no registraron a $B$. gabbi. 
CUADRO 2

\section{Especies registradas en el sendero Pájaro Sombrilla de la ReBAMB (2017-2018).}

\begin{tabular}{|c|c|c|c|c|c|}
\hline Especie/Familia & Nombre Común++ & $\begin{array}{l}\text { CITES }^{* *} / \operatorname{IUCN}^{* * *} / \\
\text { SINAC }^{* * * *}\end{array}$ & Registro seco & $\begin{array}{l}\text { Registro } \\
\text { lluvioso }\end{array}$ & Total \\
\hline Bassarycion gabbi* & Olingo & $\mathrm{A} 3 / \mathrm{LC} /-$ & 1 & o & 1 \\
\hline Canis latrans* & Coyote & -/LC/- & o & 1 & 1 \\
\hline Conepatus semistriatus & Zorro hediondo & $-/ \mathrm{LC} /-$ & 3 & 3 & 6 \\
\hline Cuniculus paca & Tepezcuinte & $\mathrm{A} 3 / \mathrm{LC} /-$ & 12 & 18 & 30 \\
\hline Dasyprocta punctata & Guatusa & A3/LC/- & o & 2 & 2 \\
\hline Dasypus novemcinctus & Armadillo & $-/ \mathrm{LC} /-$ & o & 20 & 20 \\
\hline Didelphidae + & Zorro pelón & $-/-/-$ & 18 & 26 & 44 \\
\hline Eira barbara & Tolomuco & A3/LC/- & 3 & 5 & 8 \\
\hline Herpailurus yagoarundi & Yaguarundí & $\mathrm{A} 1 / \mathrm{LC} / \mathrm{PE}$ & 5 & 3 & 8 \\
\hline Leopardus pardalis & Manigordo & $\mathrm{A} 1 / \mathrm{LC} / \mathrm{PE}$ & 15 & 20 & 35 \\
\hline Leopardus wiedii & Caucel & $\mathrm{A} 1 / \mathrm{NT} / \mathrm{PE}$ & 5 & 24 & 29 \\
\hline Mazama temama & Cabro de monte & -/DD/- & o & 1 & 1 \\
\hline Microsciurus alfari & Ardilla enana & $-/ \mathrm{LC} /-$ & o & 4 & 4 \\
\hline Mustella frenata & Comadreja & -/LC/- & o & 4 & 4 \\
\hline Nassua narica & Pizote & $-/ \mathrm{LC} /-$ & 4 & 60 & 64 \\
\hline Pecari tajacu & Saíno & A2/LC/- & 106 & 187 & 293 \\
\hline Puma concolor & Puma & $\mathrm{A} 1 / \mathrm{LC} / \mathrm{PE}$ & 10 & 24 & 34 \\
\hline Sciurus granatensis* & Ardilla roja & -/LC/- & o & 2 & 2 \\
\hline Sciurus variegatoides & Ardilla común & $-/ \mathrm{LC} /-$ & 8 & 19 & 27 \\
\hline Sylvilagus floridanus* & Conejo silvestre & $-/ \mathrm{LC} /-$ & o & 5 & 5 \\
\hline Tamandua mexicana* & Oso hormiguero & $\mathrm{A} 3 / \mathrm{LC} /-$ & 1 & 4 & 5 \\
\hline
\end{tabular}

* Nuevos registros con fotografía y video, según estudios de mamíferos (Sánchez et al, 2019).

** A1 = Apéndice uno, A2 = Apéndice dos, A3 = Apéndice tres (CITES, 2017, 2019 y 2020; La Gaceta, 1973).

*** DD = Datos deficientes, LC = Preocupación mínima, NT = Casi amenazado, EN = En peligro de extinción (IUCN, 2018).

$* * * * \mathrm{PE}=$ Peligro de extinción, según resolución R-SINAC-CONAC-o92-2017

+ Distinción taxonómica hasta familia (Didelphidae).

++Nombre común en Costa Rica (Carrillo, Wong \& Saénz, 1999, Mora, 2000).

Los índices de diversidad mostraron que, tanto en la época seca como en la lluviosa, el sendero Pájaro Sombrilla presentó una alta biodiversidad (Cuadro 3). La época seca es de cuatro meses, de enero a abril y la lluviosa de ocho, de mayo a diciembre.
El índice de Margalef indica que la diversidad es alta, debido a que los resultados se alejan de cero para ambas épocas (seca Dmg = 2.47, lluviosa Dmg = 3.09) (Cuadro 3). El índice de equidad de Pielou mostró que en ambas épocas se mantiene una abundancia de diversidad de 
media a alta $($ seca e $=0.69$. lluviosa e $=0.69)$. Finalmente, el índice de Shannon-Wiener mostró una diversidad con tendencia alta, con un índice total de $\mathrm{H}^{\prime}=2.04(\mathrm{Ln}(\mathrm{S})=$ 3.04) (Cuadro 3). Sin embargo, durante la época seca se obtuvo un índice de diversidad con un $\mathrm{H}^{\prime}=1.86$, mientras en la época lluviosa se registró un $\mathrm{H}^{\prime}=2.03$. La prueba de T modificada de Hutchenson, para una prueba de dos colas, mostró que la hipótesis nula (Ho) se acepta ya que, no se puede asegurar una diferencia entre la diversidad de la estación seca y lluviosa $(\mathrm{n}=21, \mathrm{t}=1.69 \mathrm{p}=0.05, \mathrm{gl}=619)$ (Cuadro 3).

\section{CUADRO 3}

Índices de diversidad aplicados al sendero Pájaro Sombrilla, ReBAMB (2017-2018).

\begin{tabular}{|l|l|l|l|}
\hline Índices & Lluviosa & Seca & Total \\
\hline Riqueza específica (S) & 19 & 15 & 21 \\
\hline Índice de Margalef (Dmg) & 3.09 & 2.47 & 3.10 \\
\hline Índice de Shannon (H') & 2.03 & 1.86 & 2.04 \\
\hline Equidad de Pielou (J') & 0.69 & 0.69 & 0.66 \\
\hline Índice de Simpson (D) & 0.24 & 0.26 & 0.25 \\
\hline
\end{tabular}

Curva de Acumulación de Especies: el éxito de muestreo por acumulación de especies, los modelos de Clench $(a=13.94 ; b=0.60)$ y de acumulación de especies en el programa R Studio, muestran un crecimiento de la pendiente que se ajustó a los datos, en los cuales los modelos concuerdan que la asíntota llegó a una estabilidad de especies registradas con los métodos de vídeo y foto trampeo (Fig. 3). Se registró una proporción de fauna de $95.45 \%$.

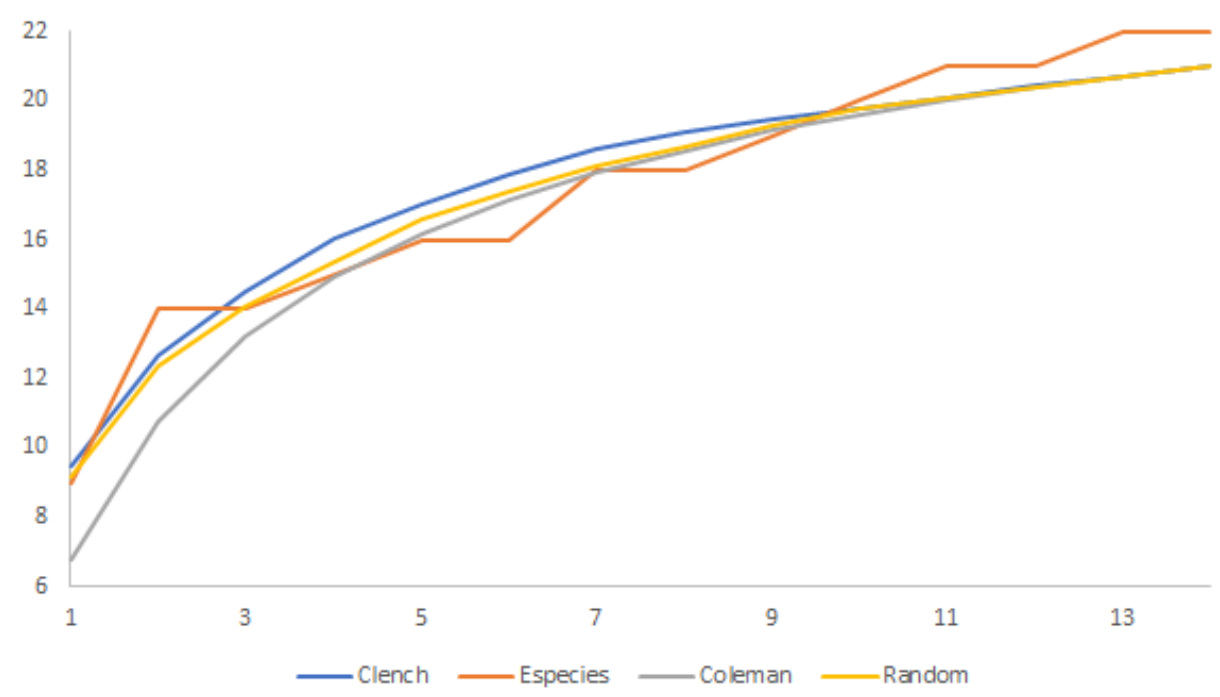

Fig. 3. Curva de acumulación de especies en el sendero Pájaro Sombrilla, (ReBAMB) bajo los modelos de Clench y acumulación de especies del paquete Vegan. 
Los resultados sugieren que para obtener un $98 \%$ de las especies se requieren de 24 muestreos, en los cuales teóricamente se deberían registrar un total de 23 especies. Lo que estadísticamente significa que, en el presente estudio, solo faltó una especie por registrar.

Patrones de Actividad: las ocho especies con registros independientes mayores o iguales a 11 fueron, $P$. tajacu, $S$. variegatoides, $L$. pardalis, $P$. concolor, $N$. narica, $D$. novemcinctus, $C$. paca, $L$ wiedii y la familia Didelphidae.
P. tajacu presentó dos picos altos de actividad, a las 6:00 a 8:00 y a las 18:00 a 20:00. Sin embargo, esta especie no tuvo acción de las 22:00 a 24:00 hasta las 4:00 a 6:00. Durante la estación seca un pico máximo de actividad fue a las 6:00 - 8:00 y otro pico alto a las 20:00 a 22:00, mientras que en la estación lluviosa sus picos de actividad fueron de 8:00 a 10:00 y de 18:00 a 20:00 (Fig. 4).

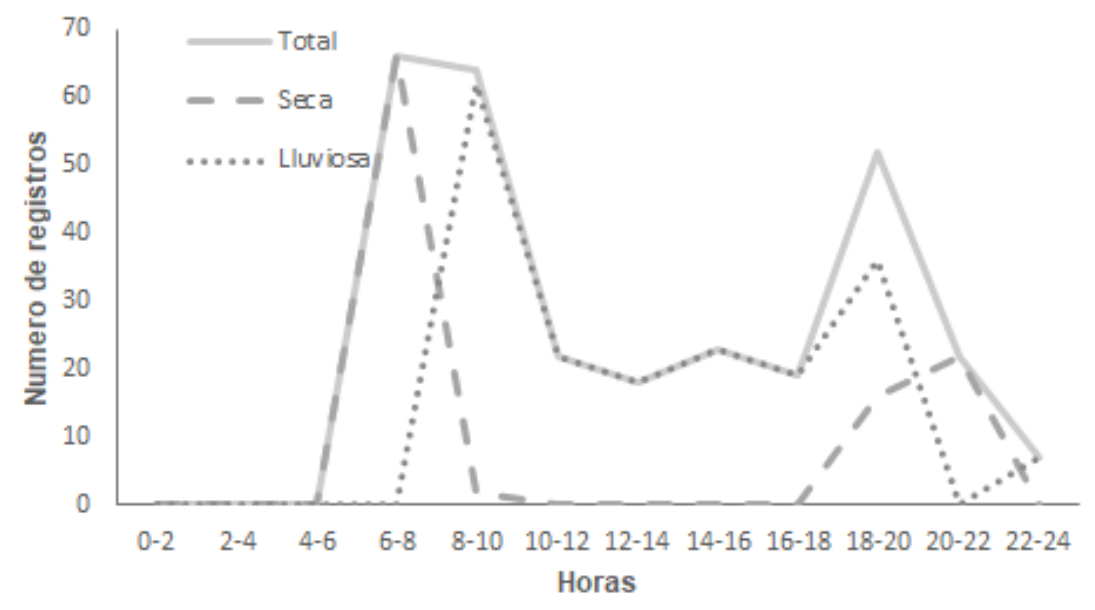

Fig. 4. Patrón de actividad del Pecari tajacu durante los distintos horarios y las estaciones seca y lluviosa, sendero Pájaro Sombrilla, ReBAMB.

Por su parte, $S$. variegatoides, presentó actividad crepuscular vespertina máxima de o6:00 a o8:00. Su actividad diurna concluyó hasta las 18:00 a 20:00 (Fig. 5).

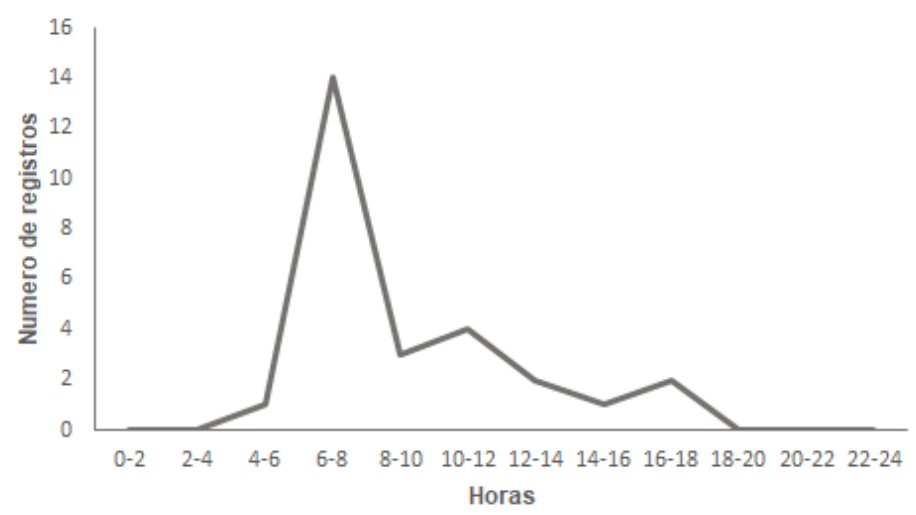

Fig. 5. Patrón de actividad del Sciurus variegatoides durante los distintos horarios, sendero Pájaro Sombrilla, ReBAMB. 
L. pardalis registró comportamiento catameral con dos picos máximos, uno de 6:00 a 8:00 y el otro de 22:00 a 24:00. Durante la estación seca destaca un periodo de inactividad de las 8:00 a 10:0o hasta las 18:00 a 20:00 (Fig. 6).

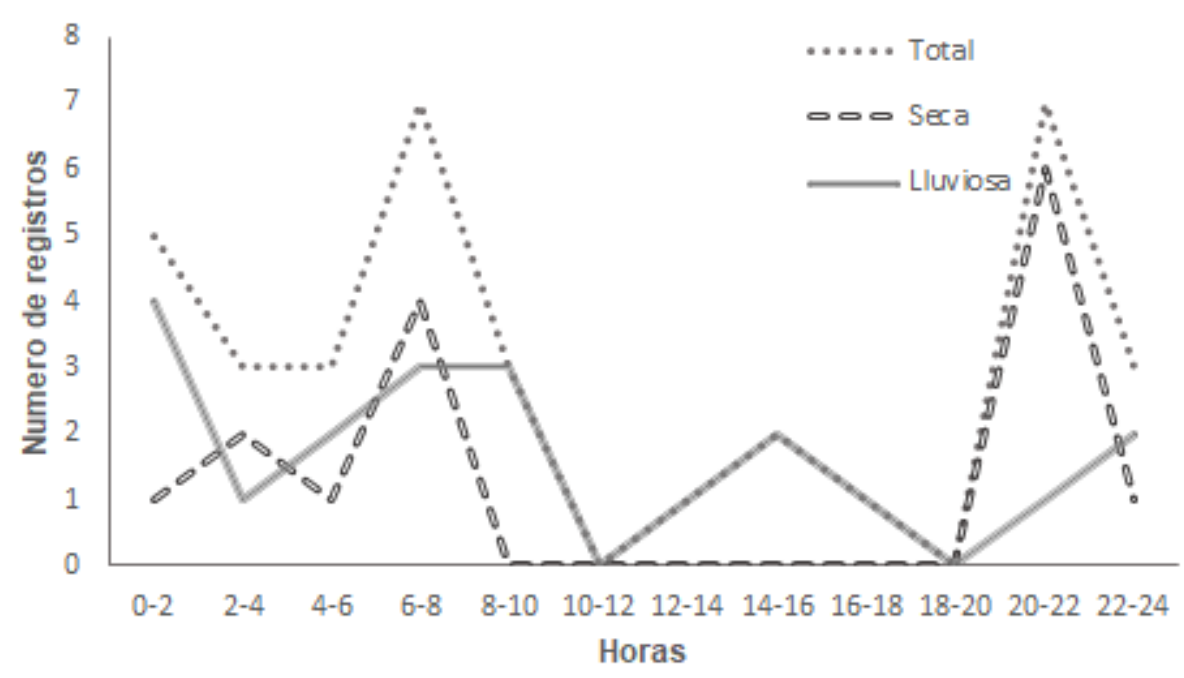

Fig. 6. Patrón de actividad de Leopardus pardalis durante los distintos horarios, y las estaciones seca y lluviosa, sendero Pájaro Sombrilla, ReBAMB.

P. concolor mostró un comportamiento catemeral con máximos de actividad entre las 14:00 a 16:00 y las 22:00 a 24:00. Durante la estación seca se redujo el número de registros, lo que representa menor actividad y presentó un periodo de inactividad de las 10:00 a 12:00 a las 14:00 a 16:00 (Fig. 7).

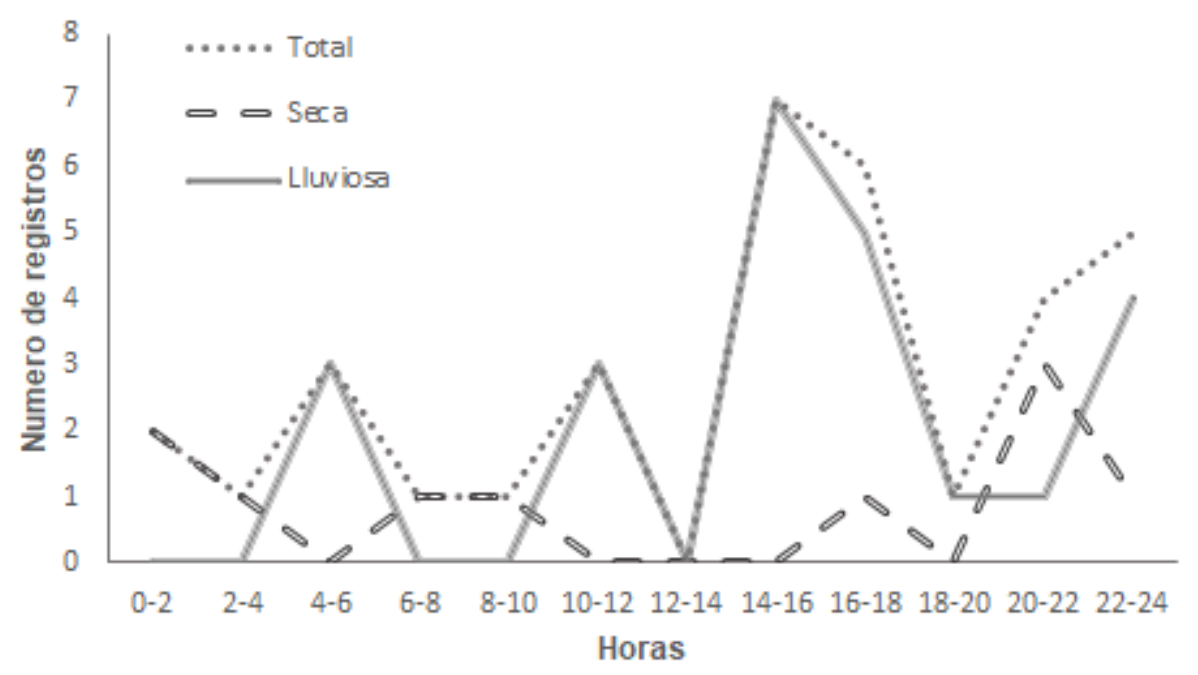

Fig. 7. Patrón de actividad del Puma concolor durante los distintos horarios, y las estaciones seca y lluviosa, sendero Pájaro Sombrilla, ReBAMB. 
N. narica exhibió actividad de las oo:0o a 02:00 a las 18:00 a 20:00 y un máximo a las 16:00 a 18:00. No obstante, el periodo de inactividad fue de las 18:00 a 20:00 a las 00:00 a 02:00 (Fig. 8).

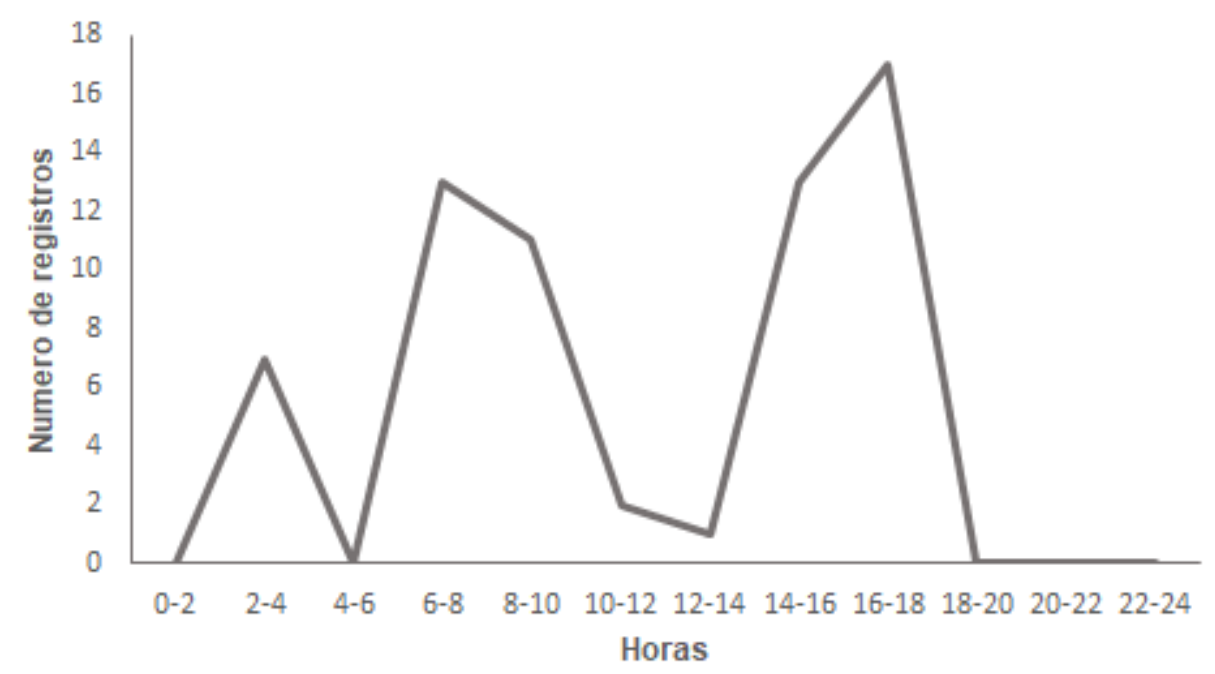

Fig. 8. Patrón de actividad de Nasua narica durante los distintos horarios, sendero Pájaro Sombrilla, ReBAMB.

D. novemcinctus siempre registró actividad excepto de 10:00 a 12:00 a las 12:00 a 14:00 y un pico máximo a las 02:00 a 04:00 (Fig. 9).

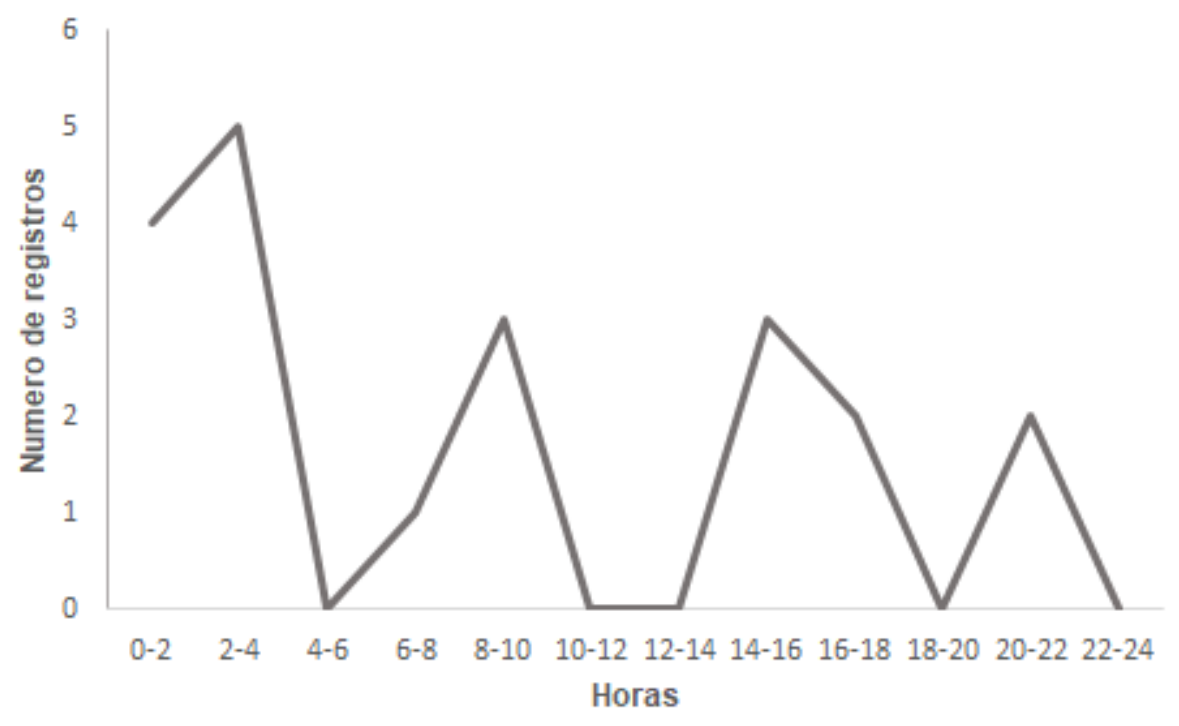

Fig. 9. Patrón de actividad de Dasypus novemcinctus durante los distintos horarios, sendero Pájaro Sombrilla, ReBAMB. 
C. paca mostró un marcado comportamiento nocturno de 16:00 a 18:00 hasta 04:00 a o6:00. Su pico máximo de actividad fue a las 20:00 a 2:00. Su actividad disminuyó durante la estación seca (Fig. 10).

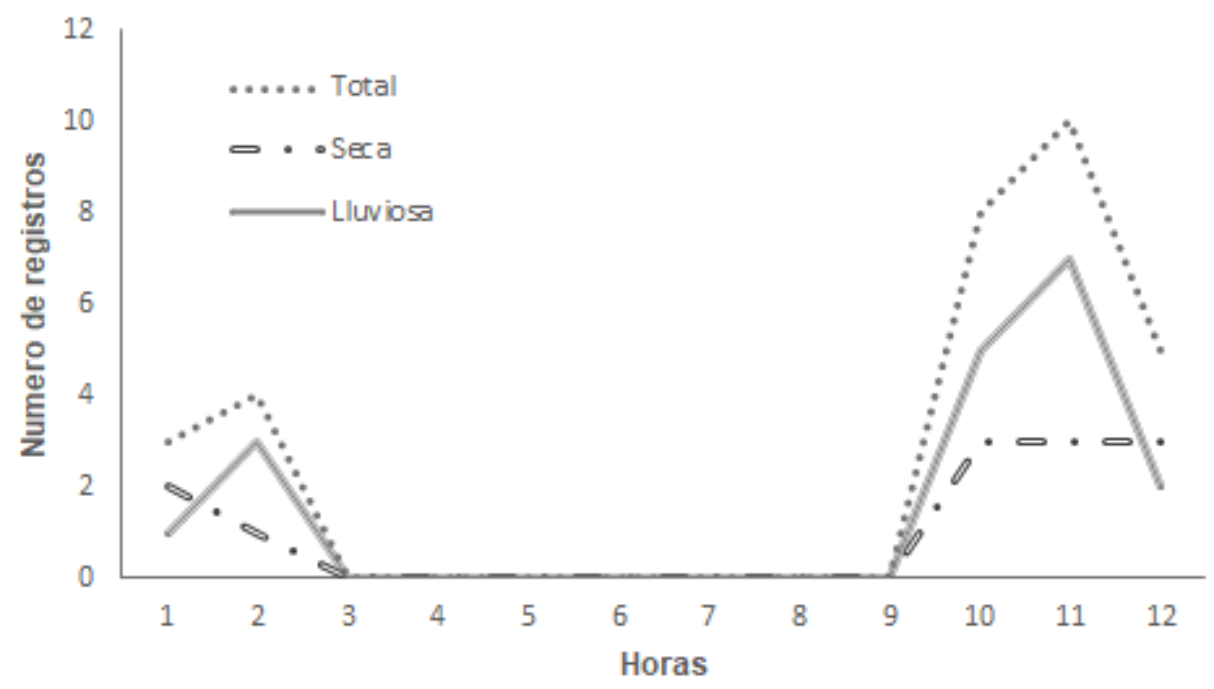

Fig. 10. Patrón de actividad de Cuniculus paca durante los distintos horarios y las estaciones seca y lluviosa, sendero Pájaro Sombrilla, ReBAMB.

L. wiedii exhibió comportamiento catameral. Esta especie tuvo un pico máximo de acción a las 2:00 a 4:0o (Fig. 11).

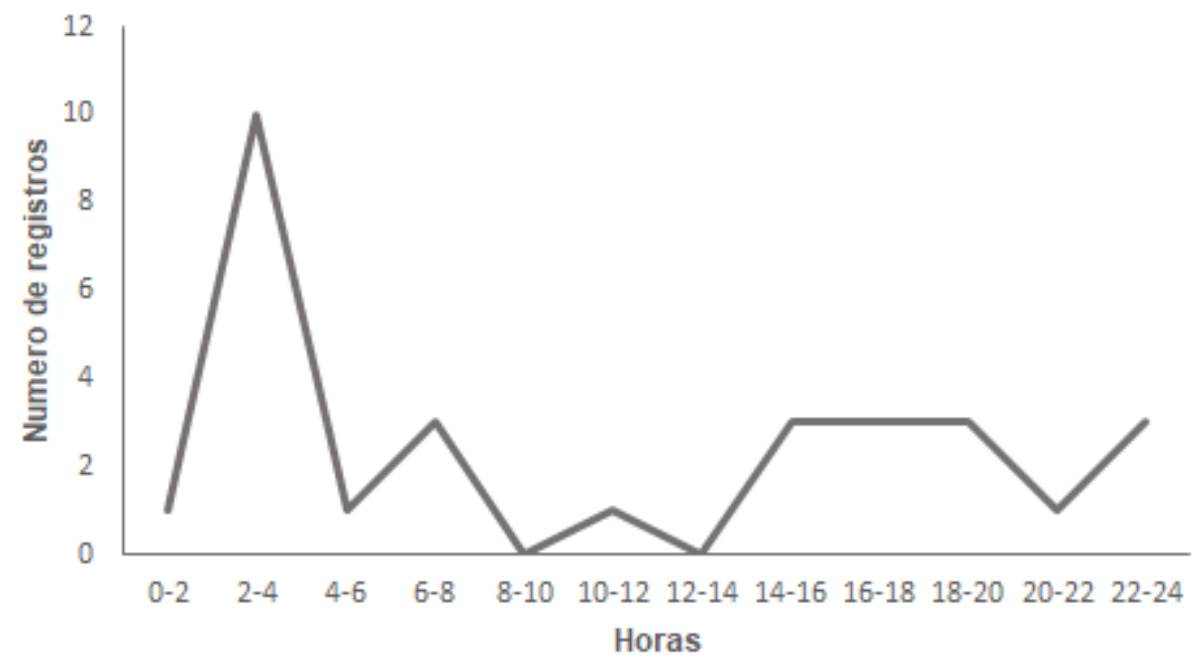

Fig. 11. Patrón de actividad de Leopardus wiedii durante los distintos horarios, sendero Pájaro Sombrilla, ReBAMB.

Por último, las especies de la familia Didelphidae mostraron un patrón de actividad nocturno de 16:00 a 18:00 hasta o6:00 a 08:00. No existió actividad de o6:00 a o8:0o hasta 16:00 a 18:00 (Fig. 12). 


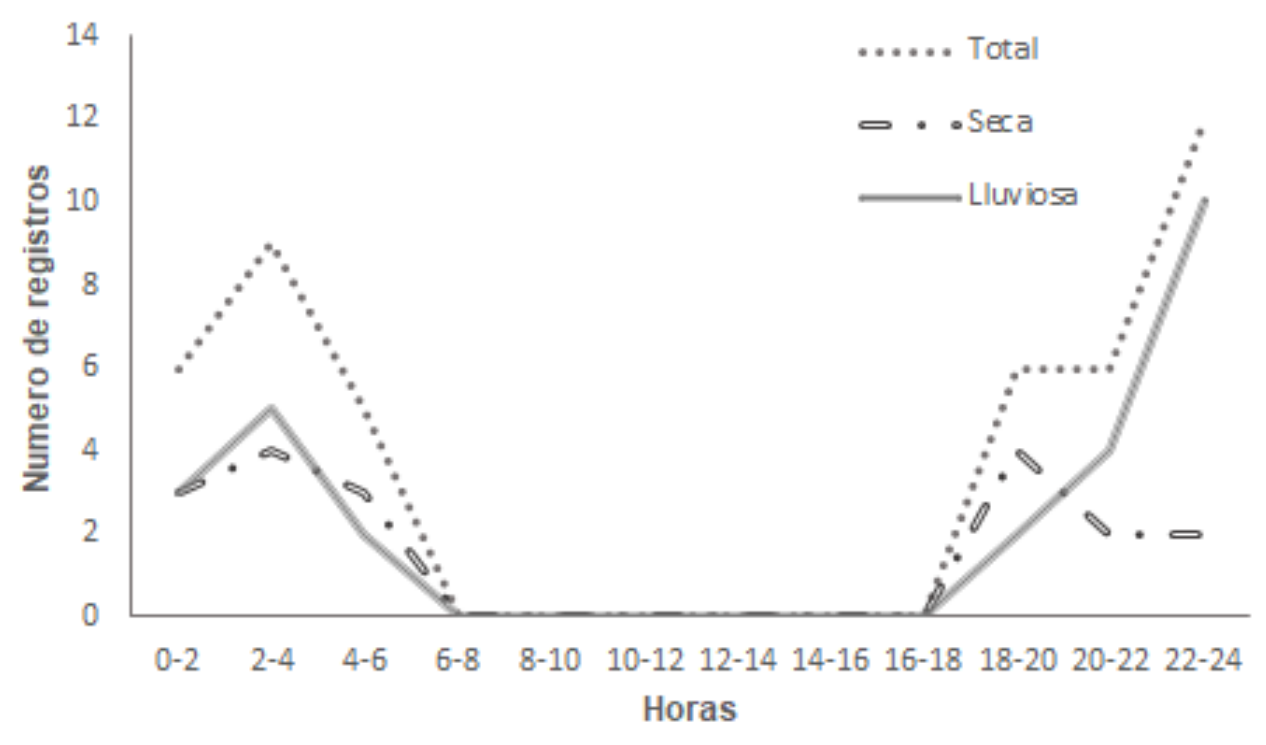

Fig. 12. Patrón de actividad de los individuos de la familia Didelphidae durante los distintos horarios y las estaciones seca y lluviosa, sendero Pájaro Sombrilla, ReBAMB.

\section{Discusión}

En cuanto a las especies con los índices de abundancia relativa más altos en este estudio, $P$. tajacu y $N$. narica, poseen un comportamiento gregario (Weldon, Cranmore \& Chatfield, 2006; García, Kahwage \& Ohashi, 2009) P. tajacu, tiende a separarse o agruparse para tolerar fragmentación, depredación y variaciones en disponibilidad de recursos (Kiltie \& Terborgh, 1983; Gómez \& Montenegro, 2012). Además, estas dos especies son generalistas u oportunistas y poseen una gran capacidad de adaptación (Hernández, Cuauhtémoc, \& List, 2018), lo que ayuda sus altos registros y observaciones en el sendero Pájaro Sombrilla. P. tajacu, es una presa del $P$. concolor cuyo índice de abundancia es relativamente alto (IAR $=2.15$, especies que conforman parte de la cadena alimenticia en el tipo de bosque tropical húmedo premontano en la ReBAMB.

La familia Didelphidae ocupó el tercer valor más alto en el IAR, esto se puede deber a que se abarcaron al menos tres especies (Didelphis marsupialis, Philander opossum y Marmosa zeledoni-mexicana) las cuales no pudieron ser claramente identificadas durante el estudio debido a la similitud entre ellas, las condiciones meteorológicas y la resolución de las cámaras durante su actividad nocturna.

Dentro de las tres especies con menor IAR se encuentran dos nuevos registros fotográficos B. Gabbi y C. latrans (Sánchez, 2000; Cartin \& Carrillo, 2017). Por su parte, C. latrans es una especie generalista poco abundante en zonas boscosas y tropicales (Servín \& Chacón, 2005). Sin embargo, en el presente estudio se realizó un registro de esta especie posterior a la Tormenta Tropical Nate, la cual provocó la caída de muchos árboles, especialmente en esta área de estudio. Lo anterior, perturbó el bosque y abrió muchos claros, lo que probablemente permitió la presencia de esta especie oportunista (Ramírez-Albores \& León-Paniagua, 2013).

La tercera especie con menor IAR registrada fue $M$. temama, Carrillo \& Sáenz (2013) mencionan que al ser presa de grandes felinos como Panthera onca y $P$. concolor, se relacionan a bosque primarios, los cuales han sufrido un declive en Costa Rica, por destrucción de hábitat y presión por cacería. Por lo anterior, estos autores sugieren, 
que esta especie puede ser indicadora para estimar la salud del ecosistema. Esta área, del presente estudio de la ReBAMB, es considerado como bosque prístino.

En esta investigación, que abarcó el área del sendero Pájaro Sombrilla se logró registrar cinco especies (C. Latrans, B. gabbi, S. granatensis, T. mexicana, S. floridanus) que no habían sido encontrados en otros estudios realizados en la ReBAMB, con esta misma metodología (Cartin \& Carrillo, 2017; Sánchez et al., 2019).

Durante el estudio de Sánchez et al. (2019) que comprendió la zona del sendero La Fila en la ReBAMB, no se registraron estas especies. Esto podría deberse a la diferencia en la disponibilidad del recurso hídrico en ambos sectores, El Sombrilla presenta una mayor cantidad de riachuelos permanentes cercanos al sendero durante los 13 meses de estudio, en comparación con el sendero La Fila, en el cual la disponibilidad del recurso hídrico es más lejano. Ejemplo de esto, es la diferencia de registros entre $C$. paca encontrados en cada estudio, los cuales están fuertemente ligados a cuerpos de agua (Aquino, Gil \& Pezo, 2009), con únicamente tres reportes en La Fila y 30 para el Pájaro Sombrilla, en ambos casos, se presentaron más reportes para la época lluviosa para el sendero Pájaro Sombrilla.

Además, el estudio de Sánchez et al., (2019) realizado en el sendero La Fila, el cual se encuentra cercano a plantaciones ornamentales ubicadas al sur, podría afectar la susceptibilidad de algunas especies, por el efecto borde. No así, en el área que abarca el sendero Pájaro Sombrilla. Por ejemplo, Daily et al. (2003) clasificaron a B. gabbi como altamente vulnerable a los hábitats alterados, especie que no se registró en el sendero La Fila.

En este estudio, se observó una hembra de T. mexicana con una cría en la espalda, en el mes de abril. Otras investigaciones sustentan como época de lactancia en el ciclo reproductivo de la especie (Linares, 1998; Genoways \& Timm, 2003; Cuarón, 2005).

De igual manera, en la investigación que comprendió toda la Reserva realizada por Cartin \& Carrillo (2017), tampoco se encontraron estas especies, debido a que este último tuvo menos días trampa (90o) en un lapso de seis meses, en comparación con el presente estudio que abarcó 1548 días trampa durante 13 meses, en un área de menor tamaño.

Durante este estudio no fue posible ver al Tapirus bairdii, sin embargo, en un muestreo previo que se realizó en el 2016 en el mismo sendero, si se logró captar esta especie en cámara trampa. Otras investigaciones (Sánchez et al., 2019; Cartin \& Carrillo, 2017) han registrado a la T. bairdii en diferentes partes de la ReBAMB.

B. gabby típicamente ocurren en bosques tropicales siempreverdes con varios estratos (Emmons \& Feer 1997; Glatston 1994; Wainwright 2002; Prange \& Prange, 2009) lo que coincide con los registros del presente estudio, donde se encuentra en la zona de vida del bosque tropical húmedo de premontano, con al menos tres estratos. No obstante, estos mismos autores señalan que su distribución altitudinal es de los 1600 a 2000 msnm, y en esta investigación el nuevo registro altitudinal de esta especie corresponde entre 1000 a $1200 \mathrm{msnm}$.

Carnivora fue el orden más representado, con 10 especies, $L$. pardalis y $P$. concolor con mayor cantidad de registros. Emmons (1987) en Perú determinó que un 92\% de las presas de $L$. pardalis eran de menos de un kilogramo $(\mathrm{Kg})$ de peso, que el 85\% de las presas de $P$. onca eran de más de $10 \mathrm{Kg}$ y que las presas de $P$. concolor estaban en el rango de 1 a $10 \mathrm{Kg}$, estos resultados concuerdan también con el principio de la capacidad de mordida para capturar presas (Chinchilla, 1997).

Lo anterior, puede reafirmar que el orden carnivora, sea el más representado, ya que se encuentran 11 especies presa dentro de estos rangos de peso (C. semistriatus, C. paca, $D$. punctata, D. novemcinctus, $M$. alfari, $M$. frenata, $N$. narica, S. granatensis, $S$, variegatoides, $S$. floridianus, $T$. mexicana). Sin embargo, algunos autores (Aranda, 2012; Ceballos et al., 2016; Luna-Baeza, 2017; Charre-Medellín et al. 2018) mencionan que $P$. concolor tienen como presa especies de más de 10kg como es el caso del P. tajacu, con la mayor cantidad de registros por especie del sendero Pájaro Sombrilla. 
Los índices de diversidad, mostraron que, en un espacio relativamente pequeño de la ReBAMB como lo es el sendero Pájaro Sombrilla (617 metros). Se encuentra una diversidad de media a alta (Cuadro 3), esto se explica por la composición en microclimas y la morfología de la cordillera que cruza de noreste a sureste nuestro país (MINAE \& IMN, 2014; Informe Ambiente, 2017), teniendo influencia de la vertiente Caribe en su mayor parte. Este corte de vertientes lo hacen altamente productivo en recurso hídrico, altas precipitaciones y como la presente investigación afirma, con alta diversidad de mamíferos.

La curva de acumulación de especies del modelo de Clench (Soberón \& Llorente, 1993) y el paquete Vegan (Oksanen et al., 2018), es recurrente en investigaciones que miden abundancia y diversidad (Escobar, 2000; Jimenez-Valverde \& Hortal, 2003; Jiménez-Valverde et al., 2004; Loayza et al., 2006; Jenks et al., 2011; Villaseñor \& Ortiz, 2014). Esencial para determinar el punto de equilibrio donde los nuevos registros llegan a disminuir y estabilizarse. El presente estudio, en el sendero Pájaro Sombrilla, se estabilizó al obtener 21 registros. Lo anterior, permitió evidenciar estadísticamente un esfuerzo de $95.45 \%$ de los mamíferos terrestres medianos y grandes presentes en este sendero, lo que refleja ser una muestra significativa estadísticamente y viable para el análisis de diversidad y abundancia relativa.

Los patrones de actividad de $S$. variegatoides coincide con otras investigaciones en cuanto a su actividad matutina vespertina (Sánchez et al., 2019). C. paca y la familia Didelphidae, reflejan su actividad nocturna crepuscular, como en otros estudios (Emmons et al. 1989; Pérez, 1992, Michalski \& Norris, 2011; Harmsen et al. 2011; Lira-Torres, Briones-Salas, 2012). L. pardalis, presentó actividad catameral (nocturno y diurno), muy diferente a estudios que infieren que su actividad es nocturna, al igual que sus principales presas, como es el caso de C. Paca (Ludlow \& Sunquist, 1987; Emmons, 1988; Crawshaw, 1995; Bustamante, 2008). Sin embargo, otras investigaciones muestran que presas menores a 10 kilogramos, son nocturnos para evitar la depredación (Monroy-Vilchis et al. 2011).
L. pardalis, P. concolor, N. narica, D. novemcinctus y $L$, wiedii, comparten patrones de actividad catamerales, igual que en el estudio realizado en el sendero La Fila (Sanchéz et al., 2019). En Colombia, N. narica fue catameral y L. wiedii, solo nocturno (Caceres-Martinez, Acevedo \& Gonzales-Valverde, 2016). Sin embargo, Estrada (2008) menciona que, para una distribución entre México y Guatemala, el P. concolor solo presentó actividad nocturna. Las especies registradas muestran que existe una variabilidad en patrones de actividad, al comparar con otras investigaciones realizadas en diferentes países (Emmons et al. 1989; Pérez, 1992, Michalski \& Norris, 2011; Harmsen et al. 2011; Lira-Torres, Briones-Salas, 2012; Caceres-Martinez, Acevedo \& Gonzales, 2016; Sanchéz et al., 2019), demostrando la diversidad y las implicaciones que pueden causar las presiones en los diferentes ecosistemas.

N. narica, es una especie que, según estacionalidad, sexo y organización social, varía su actividad. Valenzuela (2002) describe que estos factores intervienen en la actividad, un macho solitario presenta más actividad nocturna que los grupos y en época seca, recorren menos distancia y descansan más. En los registros se observa este comportamiento, ya que los grupos solo se observaron de día, mientras que los registros solitarios, presentaron actividad nocturna.

Los principales picos de actividad de las especies $P$. tajacu, S. variegatoides, L. pardalis, N. narica, y la familia Didelphidae, coinciden con los expresados por Sánchez et al. (2019) en el sendero de La Fila. La única especie que difiere en ambas investigaciones es $P$. concolor, la cual presentó su principal pico de actividad en la segunda mitad del horario diurno (12:00 - 18:00) en el sendero Pájaro Sombrilla, mientras que, en la investigación de Sánchez et al. (2019) registró baja o nula actividad en este horario. Esto podría deberse a que $P$. concolor presenta un comportamiento catameral más marcado que otras especies, tanto en esta como en otras investigaciones de monitoreo de mamíferos (Scognamillo et al., 2003; Gómez et al., 2005; Paviolo et al., 2009; Blake et al., 2012). 
El uso de cámaras trampa permitió mostrar en video diferentes especies que no habían sido registradas en otros estudios (Sánchez, 2000; Campos \& Morúa, 1991). No obstante, hacen falta otros estudios con cámaras trampa para poder documentar de manera videográfica todas las especies de mamíferos terrestres medianos y grandes, así como sus posibles índices de abundancia, riqueza y patrones de actividad.

De este modo se pueden obtener más estudios y más capturas que permitan un mejor manejo del área protegida de manera más asertiva, especialmente en los senderos, en búsqueda de un menor impacto negativo sobre la biodiversidad del área. Además, este tipo de estudios sirven de base para hacer la zonificación del área, así la administración puede determinar cuáles áreas tienen prioridad para investigación y para docencia, de acuerdo con su categoría de manejo de reserva biológica y la ley que la constituyó.

\section{Agradecimientos}

Agradecemos a la Vicerrectoría de Investigación y a la Coordinación de Investigación, Sede de Occidente de la Universidad de Costa Rica, por apoyar con el financiamiento de esta investigación. Además, a Eduardo Carrillo Jiménez del Instituto Internacional en Conservación y Manejo de Vida Silvestre (ICOMVIS), Universidad Nacional de Costa Rica, por su gran ayuda.

\section{Literatura citada}

Aquino, R., Gil, D., \& Pezo, E. (2009). Aspectos ecológicos y sostenibilidad de la caza del majás (Cuniculus paca) en la cuenca del río Itaya, Amazonía peruana. Revista peruana de biología, 16(1), 67-72.

Aranda, J.M. 2012. Manual para el rastreo de mamíferos silvestres de México. Comisión Nacional para el Conocimiento y Uso de la Biodiversidad. Distrito Federal, México.

Arita, H. \& Figueroa, F. (1999) Geographic Patterns of Body-Mass Diversity in Mexican Mammals. Oikos, 85(2), p.310.

Artavia, A. 2015. Diagnóstico de estudios con cámaras trampa en Costa Rica (1998-mayo 2015). Proyecto MAPCOBIO (SINAC, MINAE y JICA). Heredia, Costa Rica. 75 p.

Azuara, S., (2005) Estimación de abundancia de mamíferos terrestres en un área de la Selva Lacandona, Chiapas. Tesis Licenciatura. Facultad de Ciencias, UNAM.

Blake Jg, D Mosquera, Ba Loiselle, K Swing, J Guerra \& D Romo. (2012). Temporal activity patterns of terrestrial mammals in lowland rainforest of eastern Ecuador. Ecotropica 18:137-146.

Bustamante, A. 2008. Densidad y uso de hábitat por los ocelotes (Leopardus pardalis), pumas (Puma concolor) y jaguares (Panthera onca) en la parte sureste del área de amortiguamiento del Parque Nacional Corcovado, Península de Osa. Costa Rica. Tesis de Maestría. Instituto Internacional en Conservación y Manejo de Vida Silvestre.

Cáceres-Martínez, C. H., Acevedo Rincón, A. A., \& González-Maya, J. F. (2016). Diversidad y patrones de actividad de los medianos y grandes mamíferos del Parque Nacional Natural Tamá y su zona de amortiguación, Colombia. Therya, 7(2), 285-298. 
Campos, R., \& Morúa, P. (1991). Lista preliminar con anotaciones ecológicas de la mastofauna y avifauna, en la localidad de Bajo San Jorge, Reserva Forestal de San Ramón. Memoria de Investigación Reserva Forestal de San Ramón. Oficina de Publicaciones de la Universidad de Costa Rica, San José, Costa Rica, pp. 57-72.

Carrillo, E., \& Sáenz, M. S. J. (2013). 20 años de monitoreo de cinco especies indicadoras de la salud del Bosque en el Parque Nacional Corcovado, Costa Rica. Costa Rica.

Carrillo, E.; Wong, G. \& Saénz, J. (1999) Mamíferos de Costa Rica. Heredia, INBio.

Cartín, M., \& Carrillo, E. (2017) Estado poblacional de mamíferos terrestres en dos áreas protegidas de la región central occidental de Costa Rica. Revista de Biología Tropical, 65(2), pp. 493-503.

Ceballos, G. \& Oliva, G. (2005) Los mamíferos silvestres de México. México, Conabio.

Ceballos, G., H. Zarza, C. Chávez, \& J. González-Maya. (2016). Ecology and consevation of jaguars in México: state of knowledge and future challenges. Pages 273-289 in A. Aguirre and R. Sukumar, editors, Tropical conservation: perspectives on local and global priorities. Oxford University Press, Oxford.

Centro de Investigaciones Geofisicas [CIGEFI]. (2018). Estación meteorológica de la Reserva Biológica Alberto Manuel Brenes. Datos suministrados: [en línea] Universidad de Costa Rica, Costa Rica. Disponible en: [ http://www.cigefi.ucr.ac.cr/sites/ all/themes/ucr3/files/Formulario_Solicitud_ Datos_CIGEFI.pdf ]

Charre-Medellín, J.F., Rangel-Rojas, J.M., Magaña-Cota, G., Monterrubio-Rico, T.C. \& Charre-Luna, J. L. (2018). Evidence for recurrent presence of collared peccary (Pecari tajacu) population in Guanajuato, México. Western North American Naturalist. 78(1): 106-111.
Chinchilla, F. A. (1997). La dieta del jaguar (Panthera onca), el puma (Felis concolor) y el manigordo (Felis pardalis) (Carnivora:Felidae) en el Parque Nacional Corcovado, Costa Rica. Rev. Biol. Trop. 45(3):1223-1229.

Cifuentes, M., Izurieta, A., \& de Faria, H. H. (2000). Medición de la efectividad del manejo de áreas protegidas. WWF.

Colwell, R. (2006) EstimateS: Statistical estimation of species richness and shared species from samples. Versión 9.1.o. [en línea]: Viceroy. Disponible en: [ http://viceroy.eeb.uconn.edu/estimates/ ]

Convención sobre el comercio internacional de especies amenazadas de fauna y flora silvestres. (2017). Apéndices I, II y III. [en línea] CITES. Recuperado de: [https://cites.org/sites/default/files/notif/SNotif-2016-o68-A.pdf]

Convenio sobre el comercio internacional de especies amenazadas de fauna y flora silvestre (CITES), (2019). Apéndices I, II, III. [En línea]. Recuperado de: https://www.cites.org/esp/app/appendices. php.

Convenio sobre el comercio internacional de especies amenazadas de fauna y flora silvestre (CITES), (2020) Bassaricyon gabbii. [En línea]. Recuperado de: https://cites.org/eng/node/19957

Cortez-Marcial, M. (2009). Diversidad de mamíferos medianos y grandes en dos sitios con diferente grado de conservación en La Venta, Juchitán, Oaxaca. Tesis de Maestría. Centro Interdisciplinario de Investigación para el Desarrollo integral Regional Unidad Oaxaca. México, 46 p.

Cuarón, A. D. (2005). Tamandua mexicana (Saussure, 1860) oso hormiguero. Los mamíferos silvestres de México, 121-123. 
Crawshaw, P. 1995. Comparative ecology of ocelot (Felis pardalis) and jaguar (Panthera onca) in a protected subtropical forest in Brazil y Argentina. Tesis de Doctorado. Universidad de Florida. Gainesville, Florida, USA.

Daily, G. C., Ceballos, G., Pacheco, J., Suzán, G., \& SánchezAzofeifa. (2003). Countryside biogeography of neotropical mammals: conservation opportunities in agricultural landscapes of Costa Rica. Conservation biology, 17(6), 1814-1826.

Díaz-Pulido, A. y E. Payán Garrido. (2012). Manual de fototrampeo: una herramienta de investigación para la conservación de la biodiversidad en Colombia. Instituto de Investigaciones de Recursos Biológicos Alexander von Humboldt y Panthera Colombia. 32 pp.

Emmons, L. 1988. A field study of ocelots in Peru. Revue d'Ecologie de la Terre et de la Vie, 43: 133-157.

Emmons, L. H. (1987). Comparative feeding, ecology of felids in a neotropical rainforest. Behav. Ecol. Sociobiol. 20: 271-283.

Emmons, L. H., Sherman, P., Bolster, D., Goldizen, A., \& Terborgh, J. (1989). Ocelot behavior in moonlight. Advances in neotropical mammalogy, 1989, 233242.

Emmons, L., \& Feer, F. (1997). Neotropical rainforest mammals: a field guide (No. Sirsi) i9780226207193).

Escobar, S. (2000) Diversidad de coleópteros coprófagos (Scarabaeidae: Scarabaeinae) en un mosaico de hábitats en la Reserva natural Nukak, Guaviare, Colombia. Acta zoológica mexicana, (79), pp.103121.

Garcia, A. R., Kahwage, P. R., \& Ohashi, O. M. (2009). Aspectos reprodutivos de caititus (Tayassu tajacu). Embrapa Amazônia Oriental-Artigo en periódico indexado (ALICE).
Genoways, H. H., \& Timm, R. M. (2003). The xenarthrans of Nicaragua.

Glatston, A. R. (1994). The red panda, olingos, coatis, raccoons, and their relatives: status survey and conservation action plan for procyonids and ailurids. IUCN.

Gómez H,. Wallace, R,. Ayala, G \& Tejada, R. (2005). Dry season activity periods of some Amazonian mammals. Studies on Neotropical Fauna and Environment 40:91-95.

Gómez, B., \& Montenegro, O. (2012). Abundancia del pecarí de collar (Pecari tajacu) en dos áreas protegidas de la Guayana colombiana. Mastozoología neotropical, 19(2), 311-316.

Harmsen, B.J., Foster, F., Silver, S., Ostro, L \& Doncaster, C. 2011. Jaguar and puma activity patterns in relation to their main prey. Mammalian Biology 76: $320-324$.

Hernández, C. G. E. (2008). Dieta, uso de hábitat y patrones de actividad del puma (Puma concolor) y el jaguar (Panthera onca) en la selva maya. Revista Mexicana de Mastozoología (Nueva Época), 12(1), 113-130.

Hernández, J., Cuauhtémoc, C \& List, R. (2018). Diversidad y patrones de actividad de mamíferos medianos y grandes en la reserva de la biosfera La Encrucijada, Chiapas, México. Rev. Biol. Trop. 66(2): 634-646.

Hutcheson, K. (1970). A Test for Comparing Diversities Based on the Shannon Formula. Journal of Theoretical Biology, 29: 151-154

International Union for Conservation of Nature (2018). Red List of Threatened Species [en línea] IUCN Recuperado de: [www.iucnredlist.org] 
International Union for Conservation of Nature. (2016). Red List of Threatened Species [en línea] IUCN. Recuperado de: [www.iucnredlist.org]

Jenks, K. E., Chanteap, P., Damrongchainarong, K., Cutter, P., Cutter, P., Redford, T., Lynam, A.J., Howard, J. y Leimgruber, P. (2011) Using relative abundance indices from camera-trapping to test wildlife conservation hypotheses - an example from Khao Yai National Park, Thailand. Tropical Conservation Science, 4, pp.113-131.

Jiménez-Valverde, A., \& Hortal, J. (2003). Las curvas de acumulación de especies y la necesidad de evaluar la calidad de los inventarios biológicos. Revta. Ibér. Aracnol., 8, pp. 151-161.

Jiménez-Valverde, A., Cano, J. M. y Munguira, M. L. (2004) Patrones de diversidad de la fauna de mariposas del Parque Nacional de Cabañeros y su entorno (Ciudad Real, España central) (Lepidoptera, Papilionoidea, Hesperioidea). Animal Biodiversity and Conservation., 27.2, pp. 15-24

Kiltie, R. A., \& Terborgh, J. (1983). Observations on the behavior of rain forest peccaries in Perú: Why do white-lipped peccaries form herds?. Zeitschrift für Tierpsychologie, 62(3), 241-255.

La Gaceta (1973) Ley N ${ }^{\circ}$ 5605. Costa Rica, San José, 3 de marzo de 1973.

Lira-Torres, I. \& Briones-Salas, M. A. (2011) Impacto de la ganadería extensiva y cacería de subsistencia sobre la abundancia relativa de mamíferos en la Selva Zoque, Oaxaca, México. Therya, 2, pp. 217-244.

Lira-Torres, I. \& Briones-Salas, M. A. (2012) Abundancia relativa y patrones de actividad de los mamíferos de los Chimalapas, Oaxaca, México. Acta Zoológica Mexicana (ns), 28(3), pp. 566-585.
Linares, O. J. (1998). Mamiferos de Venezuela. Sociedad Conservacionista Audubon de Venezuela, Caracas, Venezuela.

Loayza, A. P., Rios, R. S., \& Larrea Alcázar, D. M. (2006) Disponibilidad de recurso y dieta de murciélagos frugívoros en la Estación Biológica Tunquini, Bolivia. Ecología en Bolivia, 41(1), pp. 7-23

Ludlow, M. E. \& Sunquist, M. E. 1987. Ecology and behavior of ocelots in Venezuela. National Geographic Research, 3: 447-461.

Luna-Baeza, F. H. (2017). Hábitat y distribución del Jabalí de Collar (Pecari tajacu). Tesis de Ingeniero Agrónomo Zootecnista. Universidad autónoma agraria Antonio Narro.

Maffei, L., Cuellar, E. \& Noss, J. (2002) Uso de trampas cámara para la evaluación de mamíferos en el ecotono Chaco-Chiquitanía. Revista Boliviana de Ecología y Conservación Ambiental, 11, pp. 55-65.

Margalef, R. (1958) Temporal succession and spatial heterogeneity in phytoplankton. In: Perspectives in Marine biology, Buzzati-Traverso (ed.), Univ. Calif. Press, Berkeley, pp. 323-347.

Medellín, R., D. Azuara, L. Maffei, H. Zarza, H. Bárcenas, E. Cruz, R. Legaria, I. Lira, G. Ramos Fernández, y Ávila, S. (2006) Censos y Monitoreo. El Jaguar Mexicano en el Siglo XXI: Situación Actual y Manejo. CONABIO-Alianza WWF TelcelUniversidad Nacional Autónoma de México. México D.F

Michalski, F., \& Norris, D. (2011). Activity pattern of Cuniculus paca (Rodentia: Cuniculidae) in relation to lunar illumination and other abiotic variables in the southern Brazilian Amazon. Zoologia (Curitiba), 28(6), 701-708. 
Ministerio de Ambiente y Energía, Instituto Meteorológico Nacional de Costa Rica. (2014). Regiones y subregiones Climáticas de Costa Rica. Recuperado el 4 de Febrero de 2020, de Instituto Meteorológico Nacional de Costa Rica, Ministerio de Ambiente y Energía. Recuperado de: https://www.imn.ac.cr/documents/10179/20909/ Regionalizaci\% $\% \mathrm{C}_{3} \% \mathrm{~B} 3 \mathrm{n}+\mathrm{clim} \% \mathrm{C}_{3} \%$ A1tica + de+Costa+Rica

Monroy-Vilchis, O., Rodríguez-Soto, C., Zarco-González, M. \& Urios, V. (2009). Cougar and jaguar habitat use and activity patterns in Central Mexico. Animal Biology, 59: 145-157.

Monroy-Vilchis, O., Zarco-González, M. M., RodríguezSoto, C., Soria-Díaz, L., \& Urios V. (2011) Fototrampeo de mamíferos, en la Sierra Nanchititla, México: abundancia relativa y patrón de actividad. Revista de Biología Tropical, 59 (1), pp.373-383.

Mora, J., (2000) Los mamíferos silvestres de Costa Rica. San José, EUNED.

Moreno, C., (2001) Métodos para medir la biodiversidad. Zaragoa, M\&T-Manuales y Tesis SEA.

Oksanen, J., Guillaume, F., Friendly, M., Kindt, R., Legendre, P., McGlinn, D., Minchin, P., O’Hara, R.,Simpson, G., Solymos, P., Stevens, M., Szoecs, E. y Wagnere, H. (2018). Community Ecology Package [en línea] cran.r-project. Recuperado de: [https://cran.r-project.org/web/packages/ vegan/vegan.pdf]

Paviolo A, Ye Di Blanco, Cd De Angelo Y Ms Di Bitetti. (2009). Protection affects the abundance and activity patterns of pumas in the atlantic forest. Journal of Mammalogy 9o:926-934.

Perez, E.M. 1992. Agouti paca. Mammalian Species 404: 1-7.

Pielou, E. C., (1966) The measurement of diversity in different types of biological collections. Journal of Theoretical Biology. 13, pp 131-144.
Prange, S., \& Prange, T. J. (2009). Bassaricyon gabbii (Carnivora: Procyonidae). Mammalian Species, (826), 1-7.

Ramírez-Albores, J. \& León-Paniagua, L. (2013). Distribucion del coyote Canis latrans) en el Continente Americano. Biocenosis, 29: 1-2.

R Development Core Team. (2008). R: A Language and Environment for Statistical Computing. $R$ Foundation for Statistical Computing, Vienna, Austria. [en línea] http://www.R-project.org. ISBN 3-900051-07-0.

Rojas-Martínez, A. \& Moreno, C. (2014). Los servicios ambientales que generan los mamíferos silvestres. PÄDI Boletín Científico de Ciencias Básicas e Ingenierías del ICBI. 2. 10.29057/icbi. v2i3.532.

Sanchez, R., Brenes, L. C., Chavarria, K. E., \& Mejías, Y. V. (2019). Diversidad y patrones de actividad de mamíferos medianos y grandes, en el sendero La Fila, Reserva Biológica Alberto Manuel Brenes, Alajuela, Costa Rica. Pensamiento Actual, 19(33), 175-189.

Rumiz, D. I. (2010). Mamíferos medianos y grandes de Bolivia: Roles Ecológicos de los Mamíferos Medianos y Grandes. Ed. Centro de Ecología Difusión Simón I. Patiño, La Paz, Bolivia. Pp 65.

Sánchez, R., (2000). Reserva Biológica Alberto Manuel Brenes, San Ramón, Alajuela, Costa Rica. Edición de Tomás Saraví. Costa Rica, SyR impresos.

Sanderson, J., (2004) Protocolo para Monitoreo con Cámaras para Trampeo Fotográfico. Tropical Ecology Assessment and Monitoring (TEAM) Initiative. The Center for Applied Biodiversity Science (CABS). USA, Conservación Internacional. 
Scognamillo D, Ie Maxit, M Sunquist \& J Polisar. (2003). Coexistence of jaguar (Panthera onca) and puma (Puma concolor) in a mosaic landscape in the Venezuelan llanos. Journal of Zoology, London 259:269-279.

Servín, J. \& Chacón, E. (2005). Coyote. In: G. Ceballos \& G. Oliva (Eds.), Los mamíferos silvestres de México (pp. 349-350). México: FCE-CONABIO.

Shannon, C. E. (1948). A mathematical theory of communication. Bell system technical journal, 27(3), 379-423

Shimwell, D. (1971) The description and classification of vegetation. London, Sidgwick \& Jackson.

Soberón, J \& Llorente, J. (1993) The Use of Species Accumulation Functions for the Prediction of Species Richness. Conservation Biology, Volume 7. No. 3 .

Surfstat. (2018) SurfStat t-distribution calculator [en línea]. Recuperado de: https://surfstat.anu.edu. au/surfstat-home/tables/t.php

Tibco Inc. (2018). STATISTICA for Windows [en línea]. Recuperado de: https://www.tibco.com/ resources/product-download/tibco-statisticatrial-download-windows

Valenzuela, D. (2002). Nasua narica (Merriam 1902). Tejón, coatí. Instituto de Biología, UNAM, México, DF, 407-410.

Villaseñor, L. \& Ortiz, E. (2014) Biodiversidad de las plantas con flores (División Magnoliophyta) en México. Revista Mexicana de Biodiversidad.

8:134-142

Wainwright, M. (2002). The natural history of Costa Rican mammals (No. 599 W42n). Zona Tropical.
Weldon, P. J., Cranmore, C. F., \& Chatfield, J. A. (2006). Prey-rolling behavior of coatis (Nasua spp.) is elicited by benzoquinones from millipedes. Naturwissenschaften, 93(1), 14-16. 